\title{
Le développement durable face à sa crise : un concept menacé, sous-exploité ou dépassé ?
}

Sustainable Development confronted with its own crisis: A Concept Under Threat, Under-Exploited or Outdated?

\section{Jacques Theys}

\section{(2) OpenEdition}

\section{Journals}

Édition électronique

URL : http://journals.openedition.org/developpementdurable/10196

DOI : 10.4000/developpementdurable.10196

ISSN : $1772-9971$

Éditeur

Association DD\&T

Référence électronique

Jacques Theys, «Le développement durable face à sa crise : un concept menacé, sous-exploité ou dépassé ? », Développement durable et territoires [En ligne], Vol. 5, nº 1 | Février 2014, mis en ligne le 04 février 2014, consulté le 30 avril 2019. URL : http://journals.openedition.org/ developpementdurable/10196; DOI : 10.4000/developpementdurable.10196

Ce document a été généré automatiquement le 30 avril 2019

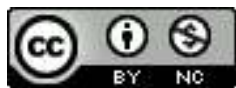

Développement Durable et Territoires est mis à disposition selon les termes de la licence Creative Commons Attribution - Pas d'Utilisation Commerciale 4.0 International. 


\title{
Le développement durable face à sa crise : un concept menacé, sous- exploité ou dépassé ?
}

\author{
Sustainable Development confronted with its own crisis: A Concept Under \\ Threat, Under-Exploited or Outdated?
}

Jacques Theys

1 Après les incertitudes des années 1990 et l'enthousiasme apparent des années 2000, le concept de développement durable est aujourd'hui incontestablement confronté à une crise. Mais, étonnamment, il y a très peu de lieux où cette situation est évoquée. Il faut donc remercier la revue Développement Durable et Territoires d'avoir pris l'initiative d'en débattre - en espérant que ce débat recevra un écho plus large dans et hors des milieux universitaires.

2 Naturellement la crise économique et les échecs successifs des conférences de Copenhague, de Rio (+20) et de Doha sont pour beaucoup dans la relative désaffection qui touche aujourd'hui l'usage de ce terme. Mais la situation économique ou géopolitique n'explique pas tout. Le changement de contexte n'a fait qu'accentuer le malaise qui, depuis les années 1980, a toujours caractérisé l'emploi de ces deux mots « développement durable». Démonétisée pour les uns - à force d'avoir été abusivement utilisée; politiquement inefficace ou même nuisible pour les autres ; l'expression semble avoir fait son temps. Et beaucoup voudraient lui substituer d'autres notions, semble-t-il plus claires, comme «l'économie verte», «la transition écologique » (ou énergétique), «la résilience » ... ou « la décroissance ». Vingt ou vingt-cinq ans après le rapport Brundtland ${ }^{1}$ , on est donc pour ce concept à un tournant qui ne met pas seulement en jeu sa crédibilité mais sans doute, en substance, sa « survie » elle-même.

3 L'intérêt d'une telle situation est qu'elle conduit, en contrepartie, à s'interroger sur ce qui fait, en profondeur, la spécificité du «développement durable »- pour peu qu'on ne le réduise pas à sa seule dimension environnementale; et sur son rôle passé dans la mondialisation des années 1990-2000, ou futur, après la crise de 2007-2008. S'il semble 
bien qu'une certaine conception du développement durable - étroitement liée au quart de siècle qui vient de s'écouler - soit historiquement dépassée, le désenchantement qu'il suscite aujourd'hui s'explique au moins autant par le fait que le concept est resté, en large partie, sous-exploité que par l'usage abusif qui en a été fait ou les résultats décevants

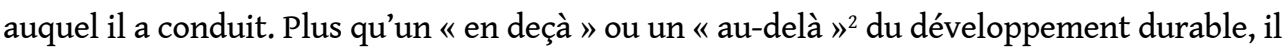
faut donc plutôt en imaginer une « seconde étape » (Theys et al., 2010).

\section{Un concept menacé, qui a épuisé les avantages de son ambiguïté}

4 En moins de cinq ans on est passé d'une époque où le « développement durable » était partout - dans les médias, les colloques scientifiques, le débat public, les processus de décision... ${ }^{3}$ - à une situation où il semble s'être effacé de la scène sociale... Entre temps il y a eu la crise de 2007-2008, l'échec de plusieurs conférences internationales, les évaluations, plutôt négatives, faites sur 25 ans de pratique, mais tous ces facteurs n'expliquent pas cette apparence de désaffection. L'expérience passée a montré que l'intérêt pour l'environnement était très sensible à la conjoncture économique; et l'assimilation qui est souvent faite entre " environnement » et " développement durable » a, naturellement, contribué à rétrograder cette préoccupation au second plan. Après la phase des " plans de relance ", les tentatives qui ont été faites pour essayer d'articuler les problématiques qui sont celles de la crise financière et celles du développement «non durable » se sont révélées infructueuses, et cet échec a aussi constitué un autre facteur d'affaiblissement ${ }^{4}$. Mais cela ne suffirait pas pour parler de crise si ne s'ajoutait à cela des raisons plus internes liées à la crédibilité du concept lui-même, et à son positionnement par rapport à d'autres apparus plus récemment - comme "l'économie verte», la "décroissance » ou « la transition écologique »... Tout se passe, en particulier, comme si l'ambiguïté, qui avait constitué dans le contexte des années 1990-2000 un facteur considérable d'attractivité du terme, était devenue aujourd'hui un de ses handicaps majeurs...

5 L'ambiguïté, on le sait, a depuis l'origine, été constitutive de l'émergence du concept de développement durable: ambiguïté dans la signification des termes (durabilité ou soutenabilité ?).et dans leur assemblage (un «oxymore»); ambiguïté dans l'origine historique (le rapport Brundtland ou bien avant...) ; ambiguïté des définitions (plus d'une centaine); confusion ou pas avec l'environnement ; ambiguïté, surtout, dans les objectifs politiques ou écologiques et dans les relations au marché ou au capitalisme mondialisé. En me centrant sur cette seule dimension politique et idéologique j'avais, il y a quatre ans dans le fil de nombreux autres travaux ${ }^{5}$ - montré que derrière le même terme on pouvait ainsi distinguer au moins six conceptions totalement différentes du développement durable (Theys, du Tertre, Rauschmayer, 2010, p. 31). (voir l'encadré 1 et les tableaux 1 et 2). Au-delà d'un noyau consensuel commun, c'est une notion qui s'est montrée, de fait, particulièrement accueillante à la diversité et à la contradiction - comme c'est le cas, d'ailleurs, pour celle de développement. Dans un article publié en 1999 pour l'Institut Européen de Florence Matthias Maier (1999) avait avancé l'idée que cette ambiguïté conceptuelle pouvait avoir trois fonctions différentes : soit constituer un habillage, une couverture, permettant les manipulations tactiques ("green washing"...) ; soit rendre possible la recombinaison des intérêts divergents, et ouvrir de nouvelles opportunités d'actions coopératives (utilisation stratégique); soit, enfin, préparer la transition vers un 
« nouveau» paradigme de développement ou de démocratie (rupture politique). Sur ce point, là aussi, toutes les interprétations sont restées ouvertes ...

Tableau 1. Des visions différentes de ce qui doit être soutenu

\begin{tabular}{|c|c|c|c|c|c|c|}
\hline & & & \multicolumn{4}{|c|}{ Conceptions } \\
\hline \multicolumn{3}{|c|}{ Questions } & $\mathbf{A}$ & B & $\mathrm{C}$ & D \\
\hline \multicolumn{3}{|c|}{ Que faut-il soutenir? } & $\begin{array}{l}\text { Le capital (humain + } \\
\text { naturel) ayant une } \\
\text { fonction économique }\end{array}$ & $\begin{array}{l}\text { Le capital naturel } \\
\text { " critique } \text { (les } \\
\text { fonctions écolog iques } \\
\text { vitales pour Phomme) }\end{array}$ & $\begin{array}{l}\text { Le capital naturel } \\
\text { «i irréversible » et } \\
\text { vulnérable }\end{array}$ & $\begin{array}{l}\text { L'ensemble du } \\
\text { patrimoine naturel } \\
\text { « signifiant } ~\end{array}$ \\
\hline \multicolumn{3}{|c|}{ Pourquoi? } & $\begin{array}{l}\text { Accroître le bien-être } \\
\text { matériel }\end{array}$ & $\begin{array}{l}\text { Accroître le bien-être } \\
\text { matériel et } \\
\text { immatériel }\end{array}$ & $\begin{array}{l}\text { Accroôtre le bien-être } \\
\text { et respecter nos } \\
\text { « obligations » par } \\
\text { rapport à la nature }\end{array}$ & $\begin{array}{l}\text { Respecter nos } \\
\text { obligations par } \\
\text { rapport à la nature }\end{array}$ \\
\hline \multirow{5}{*}{$\begin{array}{l}\text { Quelles } \\
\text { préoccupations } \\
\text { essentielles } \\
\text { (besoins)? }\end{array}$} & $\begin{array}{l}\text { Matériels humains } \\
\text { indispensables. }\end{array}$ & $\begin{array}{l}\text { Génération présente } \\
\text { Génération future }\end{array}$ & $\begin{array}{l}\mathrm{x} \\
\mathrm{x}\end{array}$ & $\begin{array}{l}\mathrm{x} \\
\mathrm{x}\end{array}$ & $\begin{array}{l}\text { Puis } \mathrm{X} \\
\mathrm{Xen} 1 \mathrm{er}\end{array}$ & $\begin{array}{l}\text { Puis } \mathrm{X} \\
\text { Puis } \mathrm{X}\end{array}$ \\
\hline & \multirow{2}{*}{$\begin{array}{l}\text { Matérielset } \\
\text { immatériels; } \\
\text { aspirations. }\end{array}$} & Génération présente & & $\mathrm{x}$ & & \\
\hline & & Génération future & & $\mathrm{x}$ & & \\
\hline & \multirow{2}{*}{ « Non humains $»$. } & Génération présente & & & Puis $\mathrm{X}$ & $\mathrm{X}$ en $1 \mathrm{er}$ \\
\hline & & Génération future & & & $\mathrm{Xen} 1 \mathrm{er}$ & $X$ en $1 \mathrm{er}$ \\
\hline \multicolumn{3}{|c|}{$\begin{array}{l}\text { Quel degré de substituabilité entre capitaux na turels/non } \\
\text { naturels ? }\end{array}$} & Considérable & $\begin{array}{l}\text { Pas entre capital } \\
\text { produit par l'homme } \\
\text { et capita Inaturel } \\
\text { critique }\end{array}$ & $\begin{array}{l}\text { Pas entre capital } \\
\text { produit par Yhomme } \\
\text { et capital naturel } \\
\text { irréversible }\end{array}$ & Très limité \\
\hline \multicolumn{3}{|c|}{ Quel niveau de soutenabilité ? } & Faible & Fort & Fort & $\begin{array}{l}\text { Extrêmement fort } \\
\text { (conservation) }\end{array}$ \\
\hline
\end{tabular}

Source : A. Dobson, 1996

Tableau 2. Derrière les mêmes mots, six conceptions très différentes (et contradictoires) du développement durable

\begin{tabular}{|c|c|c|c|}
\hline & \multicolumn{3}{|c|}{$\begin{array}{l}\text { Degrés de substituabilité entre la nature, 1'économique et le } \\
\text { social }\end{array}$} \\
\hline Options & $\begin{array}{c}\text { Soutenabilité très } \\
\text { forte } \\
\text { (très faible } \\
\text { substituabilité) }\end{array}$ & $\begin{array}{c}\text { Soutenabilité } \\
\text { forte } \\
\text { (faible } \\
\text { substituabilité) }\end{array}$ & \begin{tabular}{|c|}
$\begin{array}{c}\text { Soutenabilité } \\
\text { faible } \\
\text { (forte } \\
\text { substituabilité) }\end{array}$ \\
\end{tabular} \\
\hline $\begin{array}{c}\text { Intégration } \\
\text { économie/environnement }\end{array}$ & $\begin{array}{c}\text { a Bio-économie y et } \\
\text { décroissance durable } \\
\text { (limites absolues à la } \\
\text { croissance) }\end{array}$ & $\begin{array}{l}\text { Modernisation } \\
\text { écologique et } \\
\text { intégration } \\
\text { économie- } \\
\text { environnement } \\
\text { (stratégies } \\
\text { gagnant-gagnant, } \\
\text { économie } \\
\text { verte...) }\end{array}$ & $\begin{array}{c}\text { Croissance } \\
\text { soutenue } \\
\text { (minimisation des } \\
\text { coûts de } \\
\text { l'environnement) }\end{array}$ \\
\hline $\begin{array}{l}\text { Stratégie de } \\
\text { développement }\end{array}$ & $\begin{array}{c}\text { Économie solidaire } \\
\text { et de la «richesse }) \\
\text { (sociétés et modes de } \\
\text { développement } \\
\text { alternatifs) }\end{array}$ & $\begin{array}{l}\text { Le développement } \\
\text { durable comme } \\
\text { stratégie } \\
\text { multidimensionnel } \\
\text { le de } \\
\text { développement } \\
\text { (conception } \\
\text { spécifique du } \\
\text { développement } \\
\text { durable) }\end{array}$ & $\begin{array}{c}\text { Nouvelle } \\
\text { gouvernance ef } \\
\text { entreprise } \\
\text { citoyenne } \\
\text { (responsabilité } \\
\text { sociale des } \\
\text { entreprises, prise } \\
\text { en compte des } \\
\text { «stakeholders», } \\
\text { actions } \\
\text { citoyennes...) }\end{array}$ \\
\hline
\end{tabular}

Source : Theys, 2010 

optimistes sur les conséquences économiques ou environnementales de la mondialisation, avec aussi un certain progrès de la gouvernance mondiale, cette ambiguïté a constitué un facteur très efficace "d'attractivité » et de dynamique du concept de développement durable. Elle a ouvert la réflexion et la créativité scientifique ou technique (plus de douze mille ouvrages recensés par Amazon et un nombre incomparablement plus grand d'articles ou de guides techniques..). Permis l'agrégation, autour de ces deux mots, d'intérêts et de thématiques sans cesse plus larges. Rendu possibles des compromis pragmatiques - autour d'une culture commune minimale («les trois piliers», les stratégies gagnant-gagnant, l'environnement.....). Et sans doute favorisé une appropriation démocratique du terme, en en faisant non pas une norme absolue et définitive, mais le résultat d'un processus pragmatique de décision collective (à l'exemple des agendas 21), la résolution pratique d'une "énigme ${ }^{6}$. Edwin Zaccai a proposé le terme «d'illusion motrice» (Zaccai, 2002). Mais durant toutes les années 1990-2000, c'est bien d'abord une remarquable dynamique de changement («le moteur ») qu'a facilité l'usage du terme ambigu de " développement durable ».

Dans un contexte aujourd'hui très différent de crise économique et de désenchantement sur la gouvernance mondiale ou européenne, c'est plutôt la part d'illusion, et les aspects négatifs de l'ambiguité qui sont mis en avant - menaçant une bonne part du crédit qui s'attachait encore à la notion de développement durable avant 2008. Depuis les premières années de son émergence l'expression a toujours suscité autant de critiques que d'interprétations ou de développements multiples. Avec le changement d'époque, ce sont plutôt ces critiques qui prennent le pas, venant justifier un désir plus général de changer de vocabulaire.

Il faudrait tout un ouvrage pour présenter sérieusement toutes ces critiques, tant elles sont nombreuses... et il n'est pas possible de le faire ici. On se limitera simplement à rappeler qu'elles se situent au moins à quatre niveaux.

D'abord des critiques sémantiques sur les fonctions discursives du «syntagme » développement durable. Une très bonne illustration en est donnée dans l'article publié en décembre 2010 par Alice Krieg-Planque (2010). Au-delà des appropriations et des glissements multiples qu'elle permet ( $\mathrm{du}$ développement soutenable à la croissance durable...) l'expression, par l'usage qu'elle fait des formules «concessives » (du type: «sans pour autant »), ne serait rien d'autre qu'un moyen de neutraliser, de dépolitiser, les contradictions qu'elle est censée mettre en évidence ...

10 Ces critiques discursives font écho à celles, beaucoup plus éclatées, qui portent sur les usages politiques et idéologiques de l'expression. Ce qui est reproché au développement durable, c'est moins de porter » un projet de nature fondamentalement "socialdémocrate-écologique " (Pestre, 2011), que de couvrir, derrière une apparence scientifico-technique, une très grande duplicité idéologique: "chimère écologique malfaisante », pour les uns ${ }^{7}$; «mystification servant à préserver les intérêts des pays occidentaux, ou ceux du système marchand », pour les autres ${ }^{8}$. Entre les discours forts de "l'éco socialisme $»^{9}$ ou de la " décroissance ", d'un côté ; de la priorité à l'économie ou à la compétitivité, de l'autre ; c'est toute la rhétorique consensuelle - propre aux années 1990-2000 - « de la fin de l'histoire ${ }^{10}$, qui est, de fait, mise en doute. Un des aspects majeurs de ce soupçon concerne, naturellement, le rapport qu'entretient le développement durable avec l'environnement : cheval de Troie des écologistes pour les 
uns, il est, symétriquement, accusé de n'être qu'un outil de marketing servant, en réalité, à relativiser la gravité des problèmes d'environnement... Sous couvert de neutralité technique et scientifique, l'expression ne servirait donc, d'abord, qu'à la manipulation stratégique...

11 Du côté scientifique ou épistémologique, au contraire, la faiblesse dont est accusée le développement durable - par exemple par rapport au mot « environnement » - est d'être une notion beaucoup trop normative, et donc incertaine, pour pouvoir se prêter à l'objectivation. L'idée qu'il pourrait y avoir une "science du développement durable ", comme le proposent certains scientifiques anglo-saxons ou du Nord de l'Europe ${ }^{11}$ ne fait pas l'unanimité ; comme est d'ailleurs mis en doute le fait que le développement durable serait réellement un concept...Et beaucoup de chercheurs considèrent qu'il y a au moins autant de dangers que d'intérêt à investir sur un sujet à ce point marqué par les logiques d'action et l'ambivalence des valeurs ${ }^{12}$. C'est cette ambiguïté normative qui a, par exemple, conduit en 2006 à transformer le programme « Villes et territoires durables » en un programme "Ville et environnement », considéré comme scientifiquement beaucoup plus légitime ${ }^{13} \ldots$ 
Entre 2005 et 2008 - juste avant la conférence de Copenhague qui a marqué un coup d'arrêt aux progrès de la coopération internationale en matière d'effet de serre - pas moins de cinq revues internationales à comité de lecture se sont créées sur le thème de la "Science de la soutenabilité »: "Sustainability: Science, Practice, Policy"; "Sustainability Science"; une section dédiée à la "sustainability science" dans les "Proceedings of the National US Academy of Sciences"; " Sustainability: The Journal of Record" ; et "Consilience: the journal of sustainable development". Cette intense activité de publication s'est accompagnée, depuis cette date, par la création de plusieurs enseignements consacrés à cette science (notamment aux États-Unis, au Japon, au Canada , en Suède, mais aussi en France); l'édition de nombreux ouvrages et manifestes; ainsi que par l'organisation de colloques périodiques, soutenus par plusieurs organisations internationales dont, notamment, l'UNESCO, l'Université des Nations-Unies, ou le programme "Human Dimension of Global Change". Tout cela manifeste la volonté, qui a pris source aux États- Unis à la fin des années 1990, de replacer sur un terrain scientifique la thématique du développement durable, menacée de devenir un «slogan » sans portée réelle.

Pendant longtemps ce sont essentiellement les économistes, à la fois économistes classiques de la croissance et des ressources, mais aussi (et peut-être surtout) chercheurs travaillant dans la mouvance de l'économie écologique, qui ont cherché à conceptualiser ce qu'était le développement durable - au point que celui- ci a souvent été assimilé à l'économie des ressources. Les promoteurs d'une "Science de la durabilité "- parmi lesquels William Clark, Robert Kates et Jill Jaeger - partent plutôt de l'écologie, des sciences de la terre et des questions de risque et de vulnérabilité, pour défendre une approche holiste et systémique des relations entre système terrestre et société, qui les conduit à prendre en compte aussi les sciences humaines, mais plutôt dans une approche behaviouriste et globale complémentaire des «sciences dures». Ce qui les intéresse essentiellement c'est l'analyse des interfaces, souvent à l'échelle terrestre, entre les « systèmes humains » et les "systèmes naturels "; les questions de pressions, de limites, de résilience... La référence est constante aux travaux, qui datent des années 1970, de Holling sur la résilience des systèmes écologiques; et les réflexions s'inspirent fortement de l'ouvrage fondateur publié en 1986 - un an avant le rapport Brundtland - sous la direction de W.C. Clark et R.E. Munn, par l'IIASA et édité à Cambridge University Press: " Sustainable development of the Biosphere" (Clark, Munn, 1986).

L'économie, l'économie écologique, les sciences de la durabilité constituent autant de cadres disciplinaires indispensables pour penser et évaluer le développement durable. Peut-on pour cela parler « d'une science du développement durable ? Le colloque organisé en 1997 par Nature Science et Société, et qui a donné lieu à l'ouvrage Le développement durable, de l'utopie au concept (Jollivet, 2001), a bien montré qu'aucune science spécifique ne pouvait s'attribuer le monopole du développement durable, et que si toutes pouvaient y contribuer utilement, aucune, non plus, ne pouvait éviter qu'il subsiste dans la notion de développement une part de valeur et de choix socio-politique qui n'est pas réductible à une norme scientifique. Il peut y avoir une science de la durabilité écologique - même si l'on en connaît les difficultés pratiques de mise en œuvre. Mais la notion de "développement durable" est beaucoup plus large et ouverte, avec des composantes sociales et politiques importantes que prennent mal en compte les approches précédentes, et se laisse finalement mal enfermer dans une science et un cadre normatif uniques.

12 Ceci renvoie enfin, en miroir, aux critiques tout aussi fortes qui sont faites au développement durable, cette fois-ci du point de vue de l'action et de ses résultats - dans une perspective pragmatique. Ces critiques sont de deux ordres, et elles mettent, là 
encore, en jeu la question de l'ambiguïté. Sur le terrain, les acteurs se plaignent d'avoir à choisir entre des objectifs flous ou sans cesse redéfinis... et des injonctions bureaucratiques définies à travers une prolifération de guidelines ou d'indicateurs impossibles à respecter ${ }^{14}$. À une échelle plus large, et avec le recul historique, les évaluations a posteriori dont on dispose montrent un décalage très grand entre les espoirs qui avaient été mis dans le développement durable et les résultats réellement atteints décalage dont on ne peut dire s'il est dû à des objectifs trop vagues ou à des moyens mal adaptés ou insuffisamment mobilisés (Theys et al., 2010). Certes le développement durable reste dans les agendas ou les organigrammes, mais il n'est pas sûr, compte tenu de ce bilan et de la situation actuelle, qu'il puisse garder le statut qu'il avait souvent acquis de « référentiel stratégique »- tant l'écart entre les promesses et leur réalité est devenu béant... C'est probablement la menace la plus forte...

doute tou ces critiques, assez largement contradictoires, témoignent-elles autan de l'importance des cloisonnements politiques ou scientifiques qu'elles ne démontrent le caractère non-opératoire du concept de développement durable. Elles rendent mal justice aux acquis - sur lesquels on reviendra - de cette notion; autant sur un plan global (l'intégration du développement durable dans la culture technique)... qu'à l'échelle de programmes ou de territoires spécifiques (la ville durable, la responsabilité sociale des entreprises, le principe de précaution, etc.). Mais chacune d'elles a incontestablement une part de justification; et on comprend que leur combinaison puisse considérablement fragiliser la crédibilité de l'injonction au développement durable- dans un contexte de crise qui n'a plus rien à voir avec celui qui existait au moment où il celui-ci a émergé.

En deux mots, il n'y a plus aujourd'hui d'évidence à ce que le concept de développement durable retrouve la place qu'il avait occupé à la charnière des années 1990-2000, notamment dans les pays européens ${ }^{15}$. Les cadres et les institutions resteront, mais la dynamique semble bien avoir été cassée. Certains s'en réjouiront. Beaucoup penseront que passer à d'autres termes comme l'économie, la transition écologique ou la résilience ... ne fera que dépoussiérer un concept qui a beaucoup vieilli, et ne changera finalement rien sur le fond. Mais de même que le passage de «l'environnement » à « l'écologie », ou de « la nature » à la « biodiversité » ont eu des effets réels mal perçus, il ne faudrait pas sous-estimer les conséquences pratiques que pourrait avoir une telle substitution...

C'est d'autant plus vrai qu'après 20 ou 25 ans d'usage beaucoup des potentialités ouvertes par le concept de développement durable sont restées très largement sous exploitées ...

\section{Un concept sous-exploité}

16 Je crois avoir été le premier à utiliser ce qualificatif de "concept sous-exploité » à l'occasion du lancement du programme "Villes et territoires durables » du PUCA et du Ministère de l'écologie, en 2004 (Theys, 2004 ; Theys, 2008). À cette époque l'usage du terme était en pleine explosion, et il y avait, naturellement un paradoxe à parler de "sous-exploitation». Mais je ne faisais qu'exprimer une déception personnelle par rapport à la rupture, à la coupure épistémologique et politique radicale, que m'avait semblé être, quinze ans plus tôt, l'émergence de la notion de développement durable par rapport à celle dont j'étais familier depuis longtemps, « d'environnement »...

17 À la fin des années 1980, juste après la publication du rapport Brundtland, j'avais déjà travaillé plus de quinze ans dans le domaine de l'environnement et l'irruption dans le 
débat public du développement durable m'était apparu à la fois comme une menace potentielle pour celui-ci mais aussi comme une opportunité historique formidable (Theys, 1991), et ceci pour trois raisons :

1. D'abord parce que je m'intéressais depuis longtemps au «développement»; que j'avais accompagné les premiers pas du CIRED, créé par Ignacy Sachs autour de la notion d'écodéveloppement; et que j'étais depuis longtemps convaincu que les problèmes d'environnement devaient d'abord être pensés en ces termes ;

2. Ensuite, parce que, travaillant dans le domaine de la prospective, je ne pouvais qu'être attentif à une notion qui privilégiait les générations futures ;

3. Enfin, et surtout, parce que je me rendais compte qu'il y avait une distance quasiinfranchissable entre ceux qui assimilaient l'environnement «aux petits oiseaux $»^{16}$ et ceux qui y voyaient un problème mondial majeur... et qu'il me semblait que le développement durable pouvait être un moyen efficace pour réduire cette distance...

Mais pour que cette opportunité historique soit saisie encore fallait-il que le développement durable ne soit pas simplement un clone, un double, de l'environnement ou même de l'économie de l'environnement; qu'il ait donc une dimension spécifique forte - de manière à pouvoir s'ajouter et non se substituer à la notion d'environnement existante depuis la fin des années 1960 et la Conférence de Stockholm...

Cette conception spécifique c'est celle qui était au cœur du rapport Brundtland - qui, en deux mots, plaidait pour une véritable synergie entre les apports et les objectifs des défenseurs de l'environnement et ceux des promoteurs du développement - dans toutes ses composantes. Il est important d'en rappeler l'ensemble des caractéristiques, car bien que très largement connues, celles-ci ne sont pas toujours perçues dans leur globalité et dans toute leur richesse. Il faut en effet en distinguer, comme le détaille le tableau 3, cinq essentielles ${ }^{17}$ :

1. D'abord le souci de construire des stratégies globales et à long terme de développement dans leurs trois dimensions - économique, sociale et écologique - (par opposition à des stratégies limitées à l'environnement); ce qui suppose la capacité de co-construire ces stratégies de manière autonome et démocratique, puis de les mettre en œuvre dans la continuité (d'où la notion de transition);

2. Ensuite, la volonté de donner, dans ces stratégies, la priorité aux générations futures, et aux ressources, risques et impasses "critiques "pour ces générations. Ceci renvoie, naturellement aux questions d'énergie, de climat ou de biodiversité, en raison des irréversibilités qui s'y manifestent ; mais aussi, beaucoup plus largement, à la prévention des ruptures économiques ou sociales majeures, à la réduction des vulnérabilités "systémiques », et au maintien ou au développement des capitaux «critiques "pour les générations futures. Comme on le voit dans le tableau 3, cela inclut, par exemple, la réduction à long terme de l'excès d'endettement ou l'investissement dans les « innovations de rupture » - dès lors qu'elles sont également favorables à l'emploi et à l'environnement ;

3. En troisième lieu, une attention majeure aux problèmes d'articulation entre global et local, et donc aussi entre territoires à différentes échelles; avec, à la fois des formes originales de coopération ou de solidarité, la prise en compte des externalités, et celle des spécificités socio écologiques aux différents niveaux ;

4. Le souci, aussi, d'accorder, dans ces stratégies, une place spécifique aux besoins essentiels des populations ou des territoires les plus pauvres ou les plus vulnérables ${ }^{18}$;

5. Et enfin, dernier aspect absolument déterminant, une autre façon d'articuler les objectifs du développement, avec l'idée qu'intégrer les différentes dimensions - économiques, sociales et 
écologiques - de celui-ci ne doit pas se réduire à un simple collage de ces composantes, mais doit conduire à redéfinir les objectifs du développement lui-même.

C'est l'apport essentiel de Roberto Camagni (1998) - à partir de l'exemple de la ville durable - que d'avoir montré que les contradictions entre ces trois dimensions étroitement interdépendantes - du développement ne pouvaient, en effet, être surmontées que par un déplacement des objectifs traditionnellement assignés à chacun de ces domaines : passer de la rentabilité économique à court terme à une productivité globale à long terme (incluant les trois facteurs valeur ajoutée, emploi, ressources) ${ }^{19}$; de la recherche de l'égalité dans l'allocation des droits sociaux à une "efficacité distributive » et à une conception élargie du bien-être et de la richesse ; et enfin, de la stricte conservation écologique à une équité environnementale ou écologique intra ou inter-générationnelle (ce qui inclut la question des inégalités écologiques). Comme on le constate, tous ces objectifs expriment la volonté non pas de gommer des contradictions, mais plutôt de les affronter...

Tableau 3 : La conception spécifique du développement durable

\begin{tabular}{|c|c|c|c|}
\hline $\begin{array}{l}\text { Objectifs } \\
\text { transversaux }\end{array}$ & Dimension économique & Dimension sociale & Dimension écologique \\
\hline $\begin{array}{l}\text { De nouveaux } \\
\text { objectifs pour le } \\
\text { développement }\end{array}$ & $\begin{array}{l}\text { Accroissement de la } \\
\text { productivité globale de long- } \\
\text { terme (travail, capital, } \\
\text { ressources) et découplage } \\
\text { entre valeur ajoutée et usage } \\
\text { de ressources naturelles. }\end{array}$ & $\begin{array}{l}\text { Efficacité } \\
\text { distributive de } \\
\text { long-termer et } \\
\text { conception élargie } \\
\text { du bien-être et de } \\
\text { la richesse. }\end{array}$ & $\begin{array}{l}\text { Équité } \\
\text { environnementale } \\
\text { intra et inter } \\
\text { générationnelle } \\
\text { (sous contrainte } \\
\text { économique } \\
\text { de long-terme) }\end{array}$ \\
\hline & $\begin{array}{ll}\text { Prévention } & \text { des risques } \\
\text { systémiques } & \text { d'effondrement } \\
\text { économique. } & \end{array}$ & $\begin{array}{l}\text { Prévention des } \\
\text { risques d'implosion } \\
\text { sociale et } \\
\text { d'effondrement } \\
\text { démographique. }\end{array}$ & $\begin{array}{l}\text { Anticipation et } \\
\text { prévention des } \\
\text { risques } \\
\text { catastrophes } \\
\text { majeures ou des } \\
\text { destructions } \\
\text { irréversibles (dont : } \\
\text { climat, biodiversité... } \\
\text { ). }\end{array}$ \\
\hline $\begin{array}{l}\text { Une priorité aux } \\
\text { générations } \\
\text { futures et aux } \\
\text { ressources et } \\
\text { risques critiques } \\
\text { pour ces }\end{array}$ & $\begin{array}{l}\text { Réduction de la vulnérabilité } \\
\text { économique (ex: mono- } \\
\text { industrie) et renforcement de } \\
\text { la résilience } \\
\text { (ex : dynamique créative). }\end{array}$ & $\begin{array}{l}\text { Financement } \\
\text { soutenable des } \\
\text { «stabilisateurs } \\
\text { sociaux" et } \\
\text { renforcement de la } \\
\text { résilience } \\
\text { (autonomie, } \\
\text { réseaux sociaux...). }\end{array}$ & $\begin{array}{ll}\text { Réduction de la } & \\
\text { vulnérabilité } & \text { aux } \\
\text { risques majeurs } & \text { et } \\
\text { renforcement de la } & \text { désilience } \\
\text { ré des } \\
\text { écosystèmes. }\end{array}$ \\
\hline
\end{tabular}

générations 


\begin{tabular}{|c|c|c|c|}
\hline & $\begin{array}{l}\text { Maintien ou développement } \\
\text { des «capitaux » économiques } \\
\text { «critiques « "écologiquement } \\
\text { compatibles» (recherche et } \\
\text { formation, infrastructures, } \\
\text { tissus économiques, locaux, } \\
\text { secteurs stratégiques...). } \\
\text { Baisse de l'endettement. }\end{array}$ & $\begin{array}{l}\text { Maintien ou } \\
\text { renforcement des } \\
\text { «capitaux sociaux } \\
\text { critiques " (liens } \\
\text { sociaux essentiels, } \\
\text { patrimoines } \\
\text { culturels). } \\
\text { Renouvellement } \\
\text { démographique } \\
\text { (écologiquement } \\
\text { soutenable). }\end{array}$ & $\begin{array}{l}\text { Maintien des capitaux } \\
\text { et des ressources } \\
\text { naturelles critiques } \\
\text { (importants, } \\
\text { menacés, } \\
\text { substituables : eau, } \\
\text { sols, paysages } \\
\text { remarquables...). } \\
\text { Substitution re de } \\
\text { ressources non } \\
\text { renouvelables par des } \\
\text { ressources r } \\
\text { renouvelables. }\end{array}$ \\
\hline $\begin{array}{l}\text { Un accès moins } \\
\text { inégal aux besoins } \\
\text { essentiels res } \\
\text { populations } \\
\text { territoires les } \\
\text { plus pauvres et } \\
\text { vulnérables }\end{array}$ & 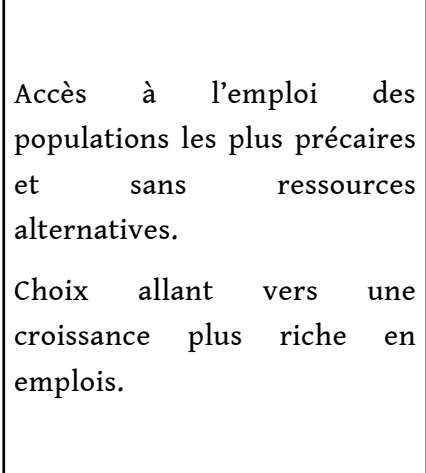 & $\begin{array}{lr}\text { Accès aux } & \text { besoins } \\
\text { essentiels r des } \\
\text { populations les plus } \\
\text { pauvres r ou } \\
\text { vulnérables. } \\
\text { Réduction rr de } \\
\text { l'écart r entre } \\
\text { besoins réels et } \\
\text { attentes } & \\
\text { subjectives. }\end{array}$ & $\begin{array}{lrr}\text { Réduction } & \text { des } \\
\text { inégalités écologiques } & \\
\text { (au } & \text { profit, } \\
\text { notamment, } & \text { des } \\
\text { populations } & \text { les } & \text { plus } \\
\text { exposées } & \text { et } & \text { ses } \\
\text { territoires } & & \\
\text { d'exclusion) } & & \end{array}$ \\
\hline \multirow{3}{*}{$\begin{array}{l}\text { Une attention } \\
\text { majeure aux } \\
\text { articulations } \\
\text { global-local et aux } \\
\text { solidarités } \\
\text { territoriales } \\
\text { (Nord-Sud) }\end{array}$} & $\begin{array}{l}\text { Accès gratuit aux biens } \\
\text { économiques } \\
\text { «communs" : information, } \\
\text { connaissances, brevets... }\end{array}$ & $\begin{array}{l}\text { Reconnaissance de } \\
\text { biens culturels ou } \\
\text { sociaux mondiaux. }\end{array}$ & $\begin{array}{l}\text { Gestion des biens } \\
\text { communs } \\
\text { écologiques, } \\
\text { mondiaux ou supra } \\
\text { territoriaux. }\end{array}$ \\
\hline & $\begin{array}{l}\text { Lutte contre le dumping fiscal } \\
\text { et les paradis fiscaux. }\end{array}$ & $\begin{array}{l}\text { Lutte contre le } \\
\text { dumping social. }\end{array}$ & $\begin{array}{l}\text { Lutte contre le } \\
\text { dumping écologique. }\end{array}$ \\
\hline & Co-développement. & $\begin{array}{l}\text { Financement } \\
\text { soutenable des } \\
\text { solidarités au profit } \\
\text { des territoires les } \\
\text { plus pauvres et } \\
\text { vulnérables. }\end{array}$ & $\begin{array}{l}\text { Réduction de } \\
\text { "l'empreinte } \\
\text { écologique " externe } \\
\text { et compensation des } \\
\text { externalités entre } \\
\text { territoires. }\end{array}$ \\
\hline
\end{tabular}

21 Au moins sur deux points cette conception spécifique se distingue très clairement de celle qui s'est affirmée au cours des 25 dernières années - d'abord dans l'opinion publique ${ }^{20}$, mais aussi dans le monde des praticiens et très largement celui de la recherche...

D'abord elle va bien au-delà de la question qui s'est posée dès Stockholm de l'intégration de l'environnement et la problématique des ressources dans l'économie, en élargissant, par exemple, les notions de risque majeur ou de vulnérabilité aux systèmes sociaux ou économiques. Elle s'écarte donc fortement de la confusion souvent faite entre environnement et développement durable... Mais cet élargissement n'infirme en rien les 
préoccupations qui sont au centre de la conception «environnementale» du développement durable : elle en renforce, au contraire, la légitimité. Parmi les risques majeurs à long terme, ceux causés à l'environnement ont, en effet, un statut spécifique, en raison de leur fréquente irréversibilité, de la faible substituabilité de beaucoup de ressources écologiques; et parce que ni la nature, ni les générations futures, ne participent directement au débat démocratique. L'environnement est aussi un domaine où il existe, par ailleurs, de fortes inégalités et où les notions de «besoins essentiels » ou de « biens communs multi échelles » sont particulièrement appropriées...

La seconde particularité, insuffisamment mise en évidence dans la conception qui s'est imposée, est qu'il ne s'agit pas seulement d'un outil d'agrégation et de coordination (« les trois... ou quatre piliers »), mais aussi d'un principe de hiérarchisation des objectifs de l'action : donner la préférence - ou au moins une forte priorité - au long terme sur le court terme, au global sur le local... mais aussi aux besoins fondamentaux non-satisfaits, aux populations et aux territoires les plus vulnérables et exposés aux inégalités, aux biens communs et aux capitaux les plus critiques (et donc, implicitement, à l'environnement). Ce n'est donc pas simplement un appel à des approches transversales, au compromis, ou aux «bons sentiments $»^{21}$, mais une injonction politique à accorder une attention prioritaire, notamment, à l'évitement des situations manifestement non durables et des « impasses ou risques majeurs pour le futur ». Au-delà d'une certaine forme de rationalité technique et scientifique, c'est aussi, en deux mots, un projet politique spécifique... qui ne se confond ni avec l'écologie politique, ni avec la croissance verte, ni avec la décroissance et doit être discuté démocratiquement comme tel...

Considérer que le concept de développement durable a été "sous-exploité», c'est simplement constater que par rapport à la richesse de cette conception spécifique, le choix a été fait d'en adopter une version plutôt pauvre, édulcorée (Brodhag, 2008) s'attachant essentiellement à intégrer l'environnement dans le marché et l'aménagement, à moderniser les formes de gouvernance dans l'entreprise ou les territoires, et à faire un "collage ", souvent rhétorique, entre les "trois piliers «du développement, avec des débats sur la nécessité d'en ajouter d'autres (culture, éthique, démocratie...). Sans doute était ce indispensable pour susciter de l'adhésion. Mais ce qui a été gagné en extension a été perdu en profondeur: les problématiques les plus innovantes sur les «capitaux critiques", la justice entre générations ${ }^{22}$, les besoins essentiels, les relations entre développement durable et formes de régulation du capitalisme, la gouvernance multi échelle et ou multi-temporelle, etc. (Zaccai et Zuindeau, 2010 ; Hamdouch et Depret, 2010 ; Emelianoff, 2011), n'ont pas été intégrées comme elle auraient dû l'être, tandis que les développement plus récents sur la résilience ou les inégalités écologiques s'en sont, en partie, détachées... En deux mots, les questions de temporalités, d'articulation avec le social ou avec les risques économiques, d'interrelations entre territoires et plus globalement de stratégies de développement, n'ont pas reçu l'attention qu'ils auraient dû avoir $^{23}$. En mettant l'accent sur la question essentielle de la durabilité écologique, on a, au passage, oublié qu'il s'agissait en fait d'une nouvelle grammaire du développement, un programme précis pour développer autrement les économies et les territoires (que l'on pense, par exemple, à la Bretagne). Or, on ne peut s'empêcher de penser que la réduction fréquente du développement durable à sa seule dimension environnementale (pour ne rien dire de l'aspect "marketing») - et son incapacité à aborder de front les conséquences sociales et économiques des politiques écologiques - ont, en définitive, contribué, paradoxalement, à maintenir l'environnement dans un statut encore marginal, 
et à le rendre très vulnérable au contexte économique. Assumer pleinement toute sa multi-dimensionalité est donc, pour lui, un enjeu majeur. Toute la question est de savoir s'il aura encore dans le futur la capacité à le faire, et donc à fédérer toutes les notions précédentes - en y trouvant une nouvelle dynamique- ou s'il est peut être considéré comme déjà dépassé...

\section{Un concept dépassé dans sa première forme, mais, dans son objet, indépassable}

Fragilisé par ses ambiguïtés, manifestement sous exploité par rapport à sa signification spécifique, le développement durable serait-il désormais dépassé ? C'est une position qui s'est exprimée avec particulièrement de force dans la série de colloques organisés en

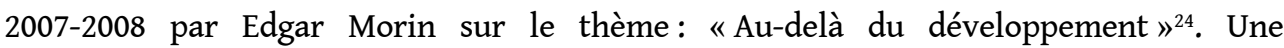
affirmation que viendrait aujourd'hui confirmer le constat d'une relative désuétude du terme - au profit d'autres plus à la mode comme "économie verte», "transition économique et sociale", "décroissance", "stratégies d'adaptation et de résilience ". Mais s'il semble bien, en effet, que, dans la forme qu'elle a prise depuis la fin des années 1980, la notion est sans doute dépassée, cela n'implique pas qu'il faille l'abandonner sans un minimum d'inventaire : plutôt que d'aller vers un « en-deçà » ou un « au-delà » de ce concept, l'idée qui sera défendue ici est qu'il faut, au contraire, en imaginer une seconde étape.

\subsection{Un concept dépassé dans sa première forme historique}

Il ne faut pas sous-estimer ce que l'histoire de ces vingt-cinq dernières années a dû à l'usage de plus en plus répandu de la notion de développement durable: une acculturation à l'environnement et à la question des ressources; une culture du compromis, de l'ouverture, de la responsabilité, du débat démocratique; une sensibilisation aux problèmes globaux et aux interdépendances territoriales ... C'est le développement durable qui, notamment, a permis dans les années 1990 de donner un second souffle à des politiques d'intégration de l'environnement et des risques dans l'économie et l'aménagement... qui étaient dans l'impasse. Ce sont, il faut l'espérer, des acquis irréversibles.

Tous ces aspects positifs n'empêchent pas, néanmoins, de penser que le concept est sans doute dépassé dans la forme historique qu'il a prise depuis la fin des années 1980 ; et ceci pour plusieurs raisons. On en a, en première partie, évoquée une première essentielle : l'excès d'ambiguïté et le fait que cette ambiguïté est de plus en plus perçue dans ses aspects négatifs (duplicité, récupération, greenwashing...). On pourra de moins en moins accepter que sous couvert de développement durable les positions les plus contradictoires soient légitimées - par exemple sur des thèmes comme l'étalement urbain ou les $\mathrm{OGM}^{25}$. Au moins comme outil de communication le discours du développement durable a déjà perdu une part importante de sa crédibilité et pourrait voir celle-ci se réduire encore beaucoup plus s'il n'arrivait pas à se renouveler. Cet aspect sémantique est naturellement important et justifie, à lui seul, pour beaucoup, un changement de 
vocabulaire. Mais il y a au moins trois autres raisons qui rendent un aggiornamento de la notion nécessaire :

1. Comme les espoirs mis en la citoyenneté globale ou les grandes régulations onusiennes, le développement durable est lié à une période historique de l'après-guerre froide et de «la mondialisation heureuse ${ }^{26}$ qui est désormais derrière nous. La crise économique, l'échec des grandes conférences internationales récentes, et le risque de marginalisation de l'Europe - qui avait en grande partie portée l'idéologie du développement durable - imposent de repenser l'usage et la signification de ce terme... pour l'adapter à un contexte économique et géopolitique qui n'a plus rien à voir avec celui dans lequel il a émergé ;

2. Une partie importante des gains que l'on pouvait attendre des stratégies gagnant-gagnant, des politiques de compromis, des améliorations incrémentales, des actions volontaires... est désormais acquise, et il est nécessaire d'imaginer d'autres logiques pour aller au-delà : dans le domaine de l'effet de serre, par exemple on peut estimer que $25 \%$ de l'effort indispensable a été ou sera rendu possible par ces stratégies coopératives ${ }^{27}$. Restent $75 \%$...

3. Enfin et surtout, il faut bien constater que dans sa version «faible » le développement durable a été impuissant à inverser et même à freiner des évolutions mondiales - et souvent locales - allant structurellement vers moins de "durabilité ». Très forte dans certaines régions, plus faible dans d'autres, la croissance des années 1990-2000 s'est faite plus que jamais en accentuant les inégalités et en augmentant les risques majeurs - sociaux, économiques ou écologiques - pour le futur. Une bonne illustration de cette vulnérabilité excessive a été la crise de 2007-2008. Mais là où il faut plus encore parler d'échec, c'est surtout dans l'incapacité à se mettre d'accord pour éviter des « impasses » irréversibles : on sait déjà, par exemple, qu'il est déjà trop tard pour empêcher qu'à la fin du siècle le réchauffement climatique soit inférieur à deux degrés, alors que c'était objectif affiché par toutes les politiques publiques depuis 20 ans... En deux mots, c'est au moment où l'expression a été la plus utilisée que le développement a été, globalement, le moins durable : le futur a été sacrifié. Avec les échecs de Copenhague et de Rio+20 et l'entrée dans une phase durable d'instabilité économique beaucoup craignent que cette inefficacité ne fasse que s'accélérer, rendant encore un peu plus illusoires les promesses que l'expression portait en elle-même...

Les quatre raisons précédentes convergent pour conclure qu'une première forme du « développement durable » est désormais dépassée. Les deux mots pourront encore rester longtemps dans les organigrammes, les titres de publication scientifiques ou les discours ; mais le diagnostic qu'il faut se résoudre à faire est - qu'au moins dans sa forme actuelle le développement durable a épuisé la majeure part de son crédit et de sa dynamique.

\subsection{Un concept indépassable : passer à une seconde étape plutôt que de changer de vocabulaire ou « fermer la parenthèse »}

29 Faire le constat de ce relatif épuisement ne veut pas dire cependant qu'il faille s'accommoder d'un changement de vocabulaire, ou abandonner le projet - à la fois politique et scientifique - qui était au cœur de la notion de développement durable, au moins dans sa conception spécifique. Parmi les options ouvertes pour surmonter la crise actuelle, il semble bien, en effet, que toutes celles qui prônent de " fermer la parenthèse " soulèvent au moins autant de problèmes qu'elles n'apportent de solutions.

Il faudrait beaucoup de pages - et ce n'est pas le lieu de la faire ici - pour discuter d'une première option qui serait celle d'une repolitisation sur un « discours fort », comme ceux que proposent les défenseurs de la décroissance » ou de « l'éco socialisme » [Löwy (2011)]. 
Sans doute ces deux courants ont-ils cette capacité à mettre dans le débat social la plupart des contradictions que le développement durable cherche, au contraire, à euphémiser : la finitude absolue de certaines ressources, la surdétermination du social et de l'écologique par l'économie, la permanence des conflits d'intérêt et des inégalités, l'absurdité de rendements du capital exigés à $15 \%$, l'épuisement - aussi - d'un certain modèle de consommation... Mais en suspendant le dépassement de ces contradictions à l'hypothèse d'une révolution improbable, et en ne proposant pas de chemin praticable, ces deux perspectives rendent finalement inaccessible l'objectif qu'elles se fixent: répondre - en urgence - à l'éventualité d'une catastrophe... C'est l'ambiguïté d'un discours fort : très mobilisateur pour les uns il sert de repoussoir pour les autres. Dans un contexte de chômage massif, où la perspective tendancielle de croissance à long terme est, au moins pour la France, voisine de 1 à $1,5 \%^{28}$, on peut craindre que l'impératif de décroissance ne décrédibilise plus qu'il ne serve les nécessités qu'il recouvre (changement des modèles de consommation et de production, mise en cause des relations entre PNB, richesse et bien être ${ }^{29} . .$. ) - et n'accroisse, en définitive, l'hostilité à l'environnement...

31 La seconde perspective serait celle d'un « retour à l'environnement » et d'un recentrage sur les disciplines scientifiques liées à cette thématique - de l'écologie à l'économie des ressources. Pour le public et une large partie de la communauté scientifique, cela n'aurait pas beaucoup d'incidences, les deux termes étant le plus souvent confondus. Dans le contexte actuel cela aurait, en revanche, comme avantage d'éviter un risque qui ne peut être écarté: que le développement durable ne soit plus désormais utilisé que pour relativiser les enjeux écologiques ou comme argument de marketing...Mais cela voudrait dire aussi renoncer à vingt-cinq années de tentatives de décloisonnement pour se replier sur un mot dont on peut se demander s'il n'est pas, lui-même, historiquement daté ...

32 Pour la plupart de ceux qui considèrent que le développement durable est dépassé, ce n'est pas ce retour à l'environnement qui est proposé, mais bien plutôt un changement de vocabulaire - un " dépoussiérage » - permettant de mettre le discours en phase avec les réalités scientifiques et politiques du moment. L'expression n'est plus à la mode et peu à peu s'imposent d'autres thématiques comme «l'économie (ou la croissance) verte », « la transition écologique et solidaire ", « la résilience » ${ }^{30}$, « la transition énergétique », « la société post carbone » (Theys, 2010 ; Vidalenc, Theys, 2013), " l'économie circulaire »,... ou la «social-écologie $»^{31} . .$. En soi, ce renouvellement de vocabulaire - faisant suite au dégonflement de la «bulle développement durable» du milieu des années 2000 - est plutôt une évolution positive tant sur le plan scientifique que politique. Il permet, en grande partie, de sortir de l'ambiguïté (même si des termes comme " économie verte » peuvent encore se prêter à beaucoup d'interprétations différentes); et témoigne d'une dynamique d'innovation très stimulante. Il faut remarquer, cependant, qu'aucune des expressions ou des concepts précédents n'est réellement substituable à celle de «développement durable ». L'économie verte, comme la résilience ou la transition écologique (ou social écologique), ne sont finalement qu'une des facettes du développement durable... Employés seuls, ils n'en constituent qu'une version appauvrie : considérer, par exemple, qu'il faille privilégier la résilience, c'est se résigner à accepter ne pas avoir de prise sur le développement futur. Aucun de ces termes n'a, non plus, la même capacité à constituer une langue commune - à mettre en tension autant d'objectifs et de mondes différents ; et donc à mobiliser autant d'acteurs... C'est la contrepartie d'un sens à chaque fois plus resserré, mais aussi plus clairement normatif (la croissance verte n'est pas la transition écologique qui n'est pas la résilience...). Le risque est de se retrouver 
dans dix ans avec une version appauvrie de l'économie verte ou de la transition énergétique, en ayant, au passage, évacué toutes les potentialités, encore largement sousexploitées, du « développement durable »...

En dehors du désir naturel de changement, il faut donc sérieusement s'interroger sur la nature du dépassement qu'induirait le passage à un l'un ou l'autre des concepts précédents. De fait, par son aptitude à mettre en connexion non seulement les finalités du développement, mais aussi les territoires et les générations, et par son souci de stratégies praticables, le développement durable est -sans doute - indépassable. Dans un contexte qui va de plus en plus être marqué par le règlement des urgences, c'est, en particulier, l'une des rares ressources susceptible d'être encore mobilisée au bénéfice des générations futures. Constater qu'une première forme est dépassée, ne veut donc pas nécessairement dire souhaiter ou accélérer sa désuétude, mais plutôt en imaginer une seconde étape. C'est la position qu'essayait d'argumenter l'article que j'avais rédigé dans l'ouvrage publié en 2010 sous le titre: Développement durable, la seconde étape (Theys, du Tertre, Rauschmayer, 2010), p. 53-60). Sans pouvoir entrer dans le détail des propositions, on peut avancer qu'une telle transformation interne supposerait, très schématiquement, au moins cinq conditions complémentaires :

- D'abord, «changer de grammaire» (et non de vocabulaire.) et revenir à la conception spécifique et multidimensionnel du rapport Brundtland (voir la partie précédente) ;

- Repenser le développement durable en fonction du nouveau contexte né de la crise (ce qui intègre les questions de transition, de résilience, d'économie verte.. mais va bien au-delà... ${ }^{32}$ ;

- Mieux distinguer dans l'action publique (et privée) ce qui relève du « vivre ensemble » et du « survivre ensemble » (ce qui suppose d'aborder de manière dissymétrique l'élaboration en commun de stratégies durables et la prévention de situations manifestement non-durables; et de traiter différemment les risques majeurs par rapport aux autres ${ }^{33}$ );

- Accorder une attention centrale aux questions de "gouvernance multi échelle», de territoires pertinents et d'articulation entre territoires - ce qui est indissociable des enjeux de fonctionnement démocratique et d'égalité territoriale, mais exige aussi et surtout de mieux articuler développement durable et mondialisation (Theys, 2013 ; Zuindeau, 2012) ;

- Et enfin, investir massivement dans la question des temporalités de l'action, des dynamiques écologiques, des inerties, des irréversibilités et des «cycles de vie » de l'innovation ou des politiques publiques.

Tout cela ne constitue, naturellement que des premières propositions qui mériteraient d'être débattues et mises à l'épreuve. Par rapport aux trois premières options évoquées, ce passage à une seconde étape du développement durable ne mettra fin, bien évidemment, ni à l'ambiguïté, ni aux déceptions, ni aux usages abusifs et manipulatoires $\mathrm{du}$ concept. Mais sans doute est-ce le prix pour que ce qui reste une innovation encore très largement sous-exploitée soit enfin pleinement valorisée, y compris par la communauté scientifique. C'est aussi la condition pour qu'après vingt-cinq ans de tâtonnements, de demi-échecs et de demi-réussites, cette « illusion motrice » devienne ce qu'elle a vocation finalement à être : le point d'appui solide et partagé d'une transition socio-écologique que chacun, partisan ou pas de l'environnement, sait, désormais, indispensable... 


\section{BIBLIOGRAPHIE}

Acquier A., 2009, « Du développement durable au développement rentable : Chronique de la marginalisation d'une démarche de développement durable dans une grande entreprise ", Gérer et comprendre, décembre, $n^{\circ}$ 98, p. 38-50.

Brodhag C., 2008, « un concept édulcoré », in Smouts M-C. (dir), Le développement durable, les termes du débat, Paris, Armand Colin, p. 89-93.

Brunel S., 2008, A qui profite le développement durable ?, Paris, Larousse.

Camagni R. 1998, «Quelles stratégies pour un développement durable des villes ? », in Villes du XXI ${ }^{\text {ème }}$ siècle, éditions du CERTU.

Clark W.C., N.M. Dickson, 2003, "Sustainability Science, The emerging research program", Proceedings of the National Academy of Science (USA), 100 (14), doi: 10.1073/pnas.1231333100

Clark W.C., R.E. Munn, 1986, Sustainable development of the Biosphere, Cambridge, Cambridge University Press.

Crifo P., M. Glachant, S. Halegatte, E. Laurent, G. Raphael, 2012, L'économie verte face à la crise, Paris, PUF.

Dobson A. 1996, "Environment Sustainabilities: An Analysis and a Typology", Environmental Politics, Vol 5, n³ , p. 401-428.

Emelianoff C., 2011, Les pouvoirs locaux dans la mondialisation écologique : remodeler l'environnement planétaire et urbain, Mémoire d'Habilitation à diriger des recherches, Université du Maine.

Fukuyama F., 1992, La fin de l'histoire et le dernier homme, Paris, Flammarion.

Giraud G., C. Renouard (dir.), 2012, Vingt propositions pour réformer le capitalisme, Nouvelle édition, Paris, Flammarion, Collection « Champs Essai ».

Godard O., 2005, Le développement durable, une chimère malfaisante ?, Chaire EDF, École polytechnique.

Gosseries A., 2004, Penser la justice entre générations. De l'affaire Perruche à la réforme des retraites, Paris, Flammarion (Aubier), Séries Alto.

Hamdouch A., Depret M-H., 2010, « Développement durable, innovations environnementales et green clusters », in Zuindeau B. (dir.), Développement durable et territoire, Villeneuve d'Ascq, Presses universitaires du Septentrion, p. 421-431.

Jollivet M. (dir.), 2001, Le développement durable : de l'utopie au concept, Paris, Elsevier, Collection « Environnement ».

Joly P-B., 2012, « Innovation responsable et développement durable : produire la légitimité des OGM et de leur monde ", Futuribles, n 383, p. 89-110.

Krieg-Planque A., 2010, « La formule « développement durable : un opérateur de neutralisation de la conflictualité », Langage et société, $\mathrm{n}^{\circ} 134$, décembre.

Laurent E., 2011, Social écologie, Paris, Flammarion.

Lévy J-P., O. Coutard (dir.), 2010, Écologies urbaines, Paris, Éditions Economica. 
Löwy M., 2011, Ecosocialisme : L'alternative radicale à la catastrophe écologique capitaliste, Paris, Éditions Mille et une Nuits.

Maier M., 1999, “The role of ideas in the politics of sustainable development", WGES Newsletter, Centre R. Schuman, European University Institute, Florence

Minc A., 1997, La Mondialisation heureuse, Paris, Plon.

Pestre D., 2011, « Développement durable : anatomie d'une notion », Natures Sciences Sociétés, Vol $19, \mathrm{n}^{\circ} 1$, Janvier-Mars.

Rotillon G., 2009, Faut-il croire au développement durable ?, Paris, L'Harmattan.

Theys J., 1991, « Histoire d'un "non-événement" : la réception du rapport Brundtland en France (1987-1991) », Actes du colloque Environnement et développement durable, Université Paris 1, Avril 1991.

Theys J., 2000, «Le développement durable et la confusion des bons sentiments », in Wachter S. et al. (dir.), Repenser le territoire, un dictionnaire critique, La Tour d'Aigues, DATAR/Éditions de L'Aube.

Theys J., 2001, « À la recherche du développement durable : un détour par les indicateurs », Jollivet M. (dir), Le développement durable, de l'utopie au concept, Paris, Elsevier, coll.

« Environnement ».

Theys J., 2004, « Le développement durable : un concept sous exploité », Contribution à l'appel d'offre du PUCA sur « Villes et territoires durables », Ministères de l'environnement et de l'équipement.

Theys J., 2008, « Le développement durable : une innovation sous exploitée », Smouts M-C. (dir), Le développement durable, les termes du débat, 2 ème édition, Paris, Armand Colin.

Theys J., 2013, « Inégalités territoriales : la perspective du développement durable », Contribution au groupe d'expert sur les inégalités territoriales mis en place par la Ministre du Logement et de l'Égalité Territoriale, février.

Theys J., C. du Tertre, F. Rauschmayer, 2010, Développement durable : la seconde étape, La tour d'Aigues, Éditions de l'Aube.

Vidalenc E., Theys J., 2013, Repenser les villes dans la société post carbone, Ministère de l'écologie, du développement durable et de l'énergie et ADEME, 300 pages, octobre 2013.

Vivien F-D., 2005, Le développement soutenable, Paris, Éditions La découverte, collection « Repères ».

Zaccai E., 2002, Le développement durable : dynamique et constitution d'un projet, Bruxelles, Éditions Peter Lang, coll. « Ecopolis ».

Zaccai E., 2011, 25 ans de développement durable : et après ?, Paris, PUF.

Zaccai E., B. Zuindeau, 2010, «Équité territoriale et développement durable », Zuindeau B. (dir), Développement durable et territoire, Villeneuve d'Ascq, Presses universitaires du Septentrion, p. 97-108.

Zuindeau B., (dir.), 2010, Développement durable et territoire, Villeneuve d'Ascq, Presses universitaires du Septentrion.

Zuindeau B., 2012, « La démondialisation pour le développement durable ? », Développement durable et territoires [En ligne], vol. 3, n 1 | Mai 2012, mis en ligne le 23 mai 2012, consulté le 15 juillet 2013. URL : http://developpementdurable.revues.org/9198 ; DOI : 10.4000/ developpementdurable.9198 


\section{NOTES}

1. Référence au colloque organisé en novembre 2008 par le Clersé : «La problématique du développement durable 20 ans après »; ainsi qu'à l'ouvrage publié Edwin Zaccai (2011).

2. Pour reprendre le titre des deux colloques organisés en 2007 et 2008 par Edgar Morin sur le thème : "Politique de civilisation : au-delà du développement durable ».

3. Selon l'enquête réalisée en juin 2008, au moment du Grenelle, par le Ministère de l'écologie $77 \%$ des personnes interrogées considéraient alors que c'était une idée à laquelle elles adhéraient et « qui avait de l'avenir ».

4. Voir, en particulier, les travaux réalisés par le groupe de prospective du Comité 21, qui se sont attachés à articuler crise économique et écologique, et les nombreux argumentaires qui ont accompagné la relance verte de 2009-2010.

5. Travaux et approches synthétisés par Franck-Dominique Vivien (2005). Voir aussi, plus spécifiquement l'article d'Andrew Dobson (1996) dont est tiré le Tableau 1.

6. Dans l'incertitude sur les préférences des générations futures, l'injonction au développement durable apparaît comme une énigme, " un concept normatif sans norme » (Theys, 2001). Franck Scherrer y voit une garantie démocratique: "l'essentiel en matière de développement durable des territoires ne tenant pas dans une forme idéale et unique mais dans la qualité et l'ouverture des processus de décision» (Colloque de la Rochelle sur les Villes du XXI ème siècle, 1998, actes publiés aux éditions du CERTU).

7. Qualificatif utilisé par J. Blamont, de l'Académie des sciences, dans : Introduction au siècle des menaces, Paris, Odile Jacob, 2004 (cité par Godard, 2005).

8. Dans de nombreux travaux et articles, Sylvie Brunel a défendu l'idée que le développement durable servait de paravent à la défense des intérêts des pays du nord, contre le sud (voir notamment Brunel, 2008). Voir aussi les critiques de Rotillon (2009).

9. Source: Löwy (2011). L'éco-socialisme, qui se situe au confluent de l'écologisme et du marxisme, ne doit pas être confondu avec la social-écologie (voir infra)...

10. Référence au livre de F. Fukuyama, qui considérait qu'après la chute du mur de Berlin toutes les sociétés partageaient désormais le même modèle fondé sur la démocratie, la culture du consensus, le libéralisme, la responsabilité... et le développement durable (Fukuyama, 1992).

11. Sur cette préoccupation de construire une véritable «science de la durabilité », voir la Revue Sustainability science; l'article collectif publié en 2001 dans la revue Science sous le titre "Sustainability Science" ( $\left.n^{\circ} 292\right)$; ou encore le programme proposé par Clark et Dickson (2003).

12. Cette distance des scientifiques par rapport à la notion de développement durable, s'exprime, par exemple, dans l'ouvrage publié sous la direction de Marcel Jollivet (2001), et en particulier dans l'article d'olivier Godard, «Le développement durable et la recherche scientifique: la difficile conciliation des logiques de l'action et de la connaissance ».

13. Les raisons de ce choix sont clairement explicitées dans l'introduction à l'ouvrage qui présente ce programme (Lévy, Coutard, 2010).

14. Une enquête publiée en 2009 par la MIT Sloan School of management ("The Business of sustainability") indiquait que pour les dirigeants d'entreprise l'ambiguïté du terme de développement durable était un des premiers obstacles à sa mise en œuvre. Dans un article de la Revue Gérer et comprendre, Aurélien Acquier (2009), décrivant la pratique d'une grande entreprise française, a bien montré comment le décalage entre la volonté stratégique de positionner l'entreprise sur le développement durable et sa traduction «bureaucratique » conduisait finalement à marginaliser cette préoccupation. 
15. Il faut rappeler que le développement durable, dans sa conception large, a d'abord été une préoccupation portée par les pays européens. Aux États-Unis, par exemple, c'est souvent l'expression de "Smart growth" qui est utilisée...

16. Lors d'un colloque organisé en 2011 sur «L'histoire du Ministère de l'environnement » l'un des membres du cabinet de G. Pompidou, créateur de ce ministère, a ainsi exprimé la surprise de constater que des préoccupations à l'origine essentiellement patrimoniales et locales avaient pu se transformer en enjeux économiques ou planétaires majeurs...

17. Toute cette partie est reprise de l'article : «Le développement durable vingt ans après: plaidoyer pour une seconde étape », (Theys in Theys, du Tertre, Rauschmayer 2010).

18. Sur la notion de besoins essentiels, voir la contribution de Felix Rauschmayer : «Qu'en est-il des besoins? Reconceptualiser le développement durable»(Theys, du Tertre, Rauschmayer, 2010).

19. Trois ans avant le rapport Brundtland, Huguette Bouchardeau, alors Ministre de l'environnement, dans l'introduction au colloque «L'environnement face à la crise », en mettant en avant cette notion de productivité globale et posant la question de l'articulation entre urgences de court terme et exigences de long terme, avait sans doute le mieux explicité cette notion de « conception spécifique » du développement durable. (Source : Cahier du GERMES n 9 , décembre 1984).

20. Toutes les enquêtes disponibles montrent que dans l'opinion publique le développement durable est d'abord associé à l'environnement (pour 65 à $80 \%$ des répondants), mais aussi au long terme. C'est, notamment le cas dans celle publiée récemment par l'ADEME (http:// www.ademe-et-vous.ademe.fr/sites/default/files/strategie-etudes/35/ademetudestrat35.pdf).

21. Qualificatif repris de l'article «Le développement durable et la confusion des bons sentiments » (Theys, 2000).

22. Parmi les nombreux travaux, peu exploités, publiés spécifiquement sur ce thème, voir, en particulier l'ouvrage d'Axel Gosseries (2004), ainsi que les publications de François Ost.

23. En particulier, l'omniprésence de la référence aux «trois piliers» a en grande partie recouvert la dimension centrale du développement durable, qui est celle de l'articulation des temporalités et de l'attention prioritaire aux générations futures.

24. Pour reprendre le titre des deux colloques organisés en 2007 et 2008 par Edgar Morin sur le thème : «Politique de civilisation : au-delà du développement durable ».

25. Allusions au colloque organisé par le Ministère du développement durable en avril 2013 sur La durabilité de la ville diffuse, ou à l'article publié par Pierre-Benoit Joly (2012), qui montre comment Monsanto a utilisé le développement durable comme principal argument de légitimation de l'usage des OGM.

26. Titre du livre publié par Alain Minc (1997).

27. Ce chiffre de $25 \%$ correspond à l'objectif fixé par l'Europe à l'horizon 2020. Au-delà de ce seuil, des ruptures beaucoup plus difficiles à faire seront nécessaires, allant très au-delà de ce qui peut être attendu des stratégies d'optimisation (Voir le numéro spécial de la revue Futuribles consacré en janvier 2013 à la « Société et à la ville post-carbone », numéro 392).

28. Dans un rapport récent sur les perspectives à long terme de croissance dans les différents pays « développés » à l'horizon 2060, l'OCDE envisage, par exemple, un taux de croissance à long terme pour la France de $1,3 \%$ (PNB par tête)...

29. Référence à l'ouvrage précurseur d'Orio Giarini (1981) Dialogue sur la richesse et le bien-être publié dans la collection des rapports au Club de Rome, suivi, ensuite, par de nombreux autres.

30. Sous l'impulsion d'institutions très actives comme le "Stockholm Resilience Center", créé en 2007, le concept de résilience a tendance, désormais, à rassembler de nombreuses thématiques auparavant intégrées sous l'égide du développement durable, et notamment celles centrées sur la dynamique des systèmes socio-écologiques. 
31. Développée par Éloi Laurent, conseiller scientifique à l'OFCE et enseignant à Stanford, la social écologie est une tentative d'articulation des objectifs de justice sociale et de soutenabilité écologique. (Laurent, 2011 ; Crifo et al., 2012).

32. Il s'agit de trouver la meilleure articulation possible entre la réponse aux urgences de court terme et la mise en œuvre de stratégies globales de durabilité à long terme, et ceci par l'innovation dans les modèles de développement et les réformes de structure. Sur ces réformes nécessaires, voir, en particulier l'ouvrage collectif coordonné par Gaël Giraud et Cécile Renouard (2012); et la contribution de C. du Tertre à Développement durable, la seconde étape (Theys, du Tertre, Rauschmayer, 2010).

33. Il y a une forte relation entre la conception spécifique de développement durable et une approche «duale»du risque qui conduit à traiter différemment les risques communs, gouvernables, et les risques critiques ou majeurs... qui ne le sont pas. Sur une typologie de ces risques majeurs, on peut se reporter soit au rapport publié par le "World Economic Forum", 2013, Global risks 2013, (vision des entreprises), soit aux nombreux articles publiés dans Science (vision des scientifiques).

\section{RÉSUMÉS}

Progressivement la notion de «Développement durable " disparaît des discours politiques et d'une partie de la littérature scientifique - au profit d'autres termes comme ceux de «transition", de « résilience », de «décroissance »... Certains lient cet effacement aux changements $\mathrm{du}$ contexte - tant le développement durable a été lié à la période de mondialisation commencée après la fin des années 1980 et achevée avec la crise de 2007-2008. D'autres y voient la conséquence logique d'une longue usure - et d'usages abusifs qui ont souvent réduit l'expression à une rhétorique creuse et finalement peu efficace... Écrit en 2012, l'article partage ces deux raisons - et en particulier la seconde: l'ambiguïé qui, à l'origine, avait largement contribué à assurer la " popularité » de ce terme a finalement conduit à en diffuser une version souvent limitée au plus petit dénominateur commun. Mais il en ajoute une troisième : si le développement durable est aujourd'hui moins attractif c'est aussi parce qu'il est resté conceptuellement sous-exploité - souvent confondu avec une version plus marchande et globale de l'environnement ou de l'économie des ressources. D'où la proposition de passer à une "seconde étape du développement durable ", mieux adaptée à la période de crise dans laquelle nous sommes entrés - et surtout redonnant à ce terme toute sa richesse et sa spécificité. Car, en définitive, les notions de transition ou de résilience, par exemple, n'en sont que deux des composantes parmi d'autres...

The concept of sustainable development seems progressively disappearing from the public and political debates, and partly from the scientific forefront - replaced by more "up to date" terms such as "resilience", "transition", "degrowth", "green economy", "smart growth"... That is, at least, the situation in France, which is not yet so much involved in the recent development of the "sustainability sciences" ongoing in the anglo-saxon countries. One possible explanation for this fading is that "sustainable development" has been deeply embedded in the context of the globalisation process which began at the end of the eighties and ended with the economic crisis in 2008. Another possible reason is the overuse - and misuse- which has been done of this badly defined notion during the last twenty years- with a weak efficiency : the ambiguity - which has 
strongly contributed to its historical success-, turns now to be a factor of distrust. This article while sharing the two previous arguments- adds to them a third one: the crisis which the concept of sustainable development is facing is also related to the fact that its potential richness or specificity has not been fully "exploited" in the past. The solution is not to "close the brackets" of "sustainable development" but to move towards "a second step" fitting more with the economic crisis and coming back to the very specificity of this concept - as it was already put forward twenty five years ago by the Brundtland report.

\section{INDEX}

Keywords : sustainable development, concept, ecological transition, resilience, sustainability science, second step

Mots-clés : développement durable, concept, transition écologique, résilience, sustainability science, deuxième étape

\section{AUTEUR}

\section{JACQUES THEYS}

Jacques Theys est enseignant à l'École des Hautes Études en Sciences Sociales, ancien responsable de la prospective au Ministère du développement durable et ancien directeur scientifique de l'Institut Français de l'Environnement. 